\title{
Investigation of the relationship between chronic montelukast treatment, asthma and depression-like behavior in mice
}

\author{
BANU CAHIDE TEL ${ }^{1 *}$, GOKCEN TELLI $^{1 *}$, SEVGEN ONDER $^{2}$, \\ EMIRHAN NEMUTLU ${ }^{3}$ and TURGUT EMRAH BOZKURT ${ }^{1}$ \\ ${ }^{1}$ Department of Pharmacology, Hacettepe University Faculty of Pharmacy; ${ }^{2}$ Department of Pathology, \\ Hacettepe University Faculty of Medicine; ${ }^{3}$ Department of Analytical Chemistry, \\ Hacettepe University Faculty of Pharmacy, Ankara, Sihhiye 06100, Turkey
}

Received January 10, 2020; Accepted June 26, 2020

DOI: $10.3892 /$ etm.2020.9459

\begin{abstract}
In 2008, the Food and Drug Administration of the US issued a warning about the neuropsychiatric side effects of montelukast. Previous clinical studies on montelukast have reported conflicting results and, to the best of our knowledge, no experimental studies concerning these side effects had been conducted. In the current study, the effect of montelukast on depression-like behavior in an ovalbumin (OVA)-induced mouse model was investigated. A total of 3 OVA challenges were applied at 2 week intervals for the persistence of asthma. Depression-like behavior was assessed using forced swim tests following each challenge and locomotor activities were evaluated using open field tests. At the end of the current study, plasma montelukast concentrations were measured and the development of asthma and effect of montelukast treatment were histopathologically examined. Inflammation scores that were increased in the OVA mice following all challenges were indicated to be reduced by montelukast treatment. The immobility time of mice increased beginning with the first challenge and this was also reduced by montelukast treatment. Montelukast administration to the control mice did not alter immobility times. Moreover, motor activity of the OVA and montelukast-treated mice were not altered. The results indicated there was no association between chronic montelukast treatment and depression. Furthermore, the chronic administration of montelukast to non-asthmatic mice did not increase immobility. However, depressive behavior increased at all time points in the OVA mice. These results indicated that chronic montelukast treatment is not associated
\end{abstract}

Correspondence to: Dr Banu Cahide Tel, Department of Pharmacology, Hacettepe University Faculty of Pharmacy, Ankara, Sihhiye 06100, Turkey

E-mail: banutel@hacettepe.edu.tr

${ }^{*}$ Contributed equally

Key words: montelukast, asthma, ovalbumin, forced swim test, depression, neuropsychiatric side-effects with depression-like behavior and confirmed the association between asthma and depression. Further studies are required to provide an improved understanding of the neuropsychiatric side effects of montelukast.

\section{Introduction}

Asthma is a common chronic inflammatory airway disease that is accompanied by respiratory symptoms, including difficulty breathing, chest tightness, wheezing and airway hyperresponsiveness (1). Globally, >300 million patients suffer from asthma and the prevalence of this disease is increasing (2-4). Montelukast is a selective leukotriene receptor-1 antagonist (LTRAs) and is widely used for the treatment of asthma (5-7). The Global Initiative for Asthma guidelines recommend LTRAs as a second-line alternative medication to inhaled corticosteroids (ICS) $(8,9)$. In children, montelukast is preferred due to the advantages of its pharmaceutical form compared with ICS (7). However, certain post-marketing studies have indicated that chronic montelukast treatment may be linked to neuropsychiatric disorders, including aggressive behavior, sleep disorders and depression (5-9). In 2008, due to the increase of these reports, the Food and Drug Administration of the US (FDA) issued a warning and indicated the enhanced risk of neuropsychiatric events associated with the use of antileukotriene agents (10). Previous clinical studies have reported an increase in suicidal tendency, depression, hallucinations and nightmares following the administration of montelukast $(5,6)$. In 2009, the prescribing information of Singulair ${ }^{\circledR}$ (montelukast sodium) was revised and the warnings and precautions section was edited to include neuropsychiatric events $(11,12)$. However, despite the results of important case reports and the warning by the FDA, no sufficient data was found to demonstrate an association between montelukast and depression $(13,14)$. Furthermore, to the best of our knowledge, no experimental studies have been conducted to investigate the association between depression-like behavior and montelukast treatment. Furthermore, asthma is considered to be a risk factor for depression (15-17). The association between asthma and depression has been examined by numerous studies and the results have demonstrated a high comorbidity between the two diseases (18-21). Depressive disorders have been reported 
to have almost 2-fold higher prevalence in young asthmatic patients compared with young non-asthmatics (22). The current study aimed to investigate the possible association between chronic montelukast treatment and depression-like behavior, and the effect of asthma on depression-like behavior in mice.

\section{Materials and methods}

Animals. A total of 130 Swiss mice (male, 65; female, 65; age, 8-12 weeks; weight, 20-25 g) were purchased from Kobay D.H.L. A.S. and were used in all experiments. Equal numbers of male and female mice were used in each experimental group. The mice were housed in a room at constant humidity and temperature $\left(22 \pm 1^{\circ} \mathrm{C}\right)$ with $12 \mathrm{~h}$ light/dark cycles and provided with food and water ad libitum. All experiments were carried out according to the EU Directive 2010/63/EU (23) for animal experiments and approved by the Animal Experimentations Local Ethics Board of Hacettepe University, Ankara, Turkey (approval no. 2018-3/2; updated by committee in the meeting commencing in 2020 for in vivo analysis of airway function, approval no. 2020-3/12).

Drug administration and experimental design. The mice were divided into four groups for the experimental asthma and forced swim test (FST) as follows: i) Control group ( $n=24$ for $1 \mathrm{st}, \mathrm{n}=18$ for $2 \mathrm{nd}$ and $\mathrm{n}=12$ for 3 rd FST); ii) ovalbumin (OVA; $n=24$ for 1 st, $n=18$ for 2 nd and $n=12$ for 3 rd FST); iii) montelukast-treated controls ( $n=18$ for 2 nd and $n=12$ for 3rd FST); and iv) montelukast-treated OVA ( $\mathrm{n}=18$ for 2 nd and $\mathrm{n}=12$ for 3 rd FST). Montelukast treatment was started after inflammation was occurred with the first challenge. In the first FST, mice were administrated montelukast for only 1 day, as the primary aim of the current study was to investigate the chronic effects of montelukast. The first FST was not performed in montelukast-treated groups. (Fig. 1). Following each FST, 6 mice were euthanized for histopathological analysis. In the treatment groups (montelukast-treated OVA and montelukast-treated control groups), montelukast (Abdi Ibrahim Pharmaceuticals; $20 \mathrm{mg} / \mathrm{kg} /$ day) was administrated orally through the drinking water every day at the same hour, starting on the first day after the first OVA challenge and montelukast treatment was continued for 20 days for 2 nd challenge and 40 days for 3rd challenge (Fig. 1). Additional groups were used for the open field test ( $\mathrm{n}=10 /$ group) and the in vivo assessment of airway function ( $n=8 /$ group). Open field tests were performed using three other groups of mice: i) The OVA group; ii) the montelukast-treated (20 days) control group; and iii) the control group. In vivo assessment of airway function was performed in another set of OVA and control groups.

Experimental asthma. An experimental asthma model was established using OVA (Sigma-Aldrich; Merck KGaA) in the OVA and montelukast-treated OVA groups (24). The OVA-induced asthma model was modified to extend the duration of asthma to two months. The mice were sensitized on days 1 and 8 by an intraperitoneal injection of $10 \mu \mathrm{g}$ OVA dissolved in $200 \mu \mathrm{l}$ PBS (Sigma-Aldrich; Merck KGaA) containing $1 \mathrm{mg}$ aluminum hydroxide (Sigma-Aldrich; Merck KGaA). Airway inflammation was induced by intranasal
OVA-challenges (0.25 $\mu \mathrm{g}$ OVA in $25 \mu \mathrm{l}$ PBS) on days 15,16 and 17 (1st challenge), 34, 35 and 36 (2nd challenge) and 53, 54 and 55 (3rd challenge) to induce and maintain chronic airway inflammation. The control groups were sensitized with OVA (0.25 $\mu \mathrm{g}$ OVA in $25 \mu \mathrm{l}$ PBS) and challenged with intranasal PBS (25 $\mu \mathrm{l}$ PBS; Fig. 1). A stratified design was used for the experiments and, therefore, equal numbers of animals from each group were used on the same days for all experiments.

In vivo assessment of airway function. Airway function was assessed in the OVA and control groups by evaluating the changes in airway resistance using the Buxco Finepoint Resistance and Compliance System (Buxco Electronics, Inc.). Testing was performed one day following the 1st OVA challenge. Mice were anesthetized with $100 \mathrm{mg} / \mathrm{kg}$ ketamine $/ 10 \mathrm{mg} / \mathrm{kg}$ xylazine intraperitoneally, tracheally cannulated and placed into individual plethysmography chambers (Buxco Electronics, Inc.). The lungs of the mice were mechanically ventilated at a respiratory rate of 150 breaths/min with a tidal volume of $200 \mu \mathrm{l}$ and a positive end expiratory pressure of $2 \mathrm{cmH}_{2} \mathrm{O}$. The basal airway resistance was recorded for $2 \mathrm{~min}$ and PBS was used for control responses. Methacholine $(1.5-48 \mathrm{mg} / \mathrm{ml})$ was administered intratracheally to measure the dose-dependent increase in respiratory resistance using an ultrasonic nebulizer (Buxco Electronics, Inc.). The maximum value measured following each dose for respiratory resistance was used as the measure of response. Airway resistance $\left(\mathrm{cmH}_{2} \mathrm{O} . \mathrm{s} / \mathrm{ml}\right)$ results were normalized to values measured following PBS nebulization.

Forced swim test. A modified version of Porsolt's FST was performed to assess the effect of montelukast treatment on depression-like behavior and repeated following each challenge $(25,26)$. Briefly, glass cylinders (height, $19 \mathrm{~cm}$; diameter, $12 \mathrm{~cm}$ ) were filled with $25 \pm 1^{\circ} \mathrm{C}$ water to a $15 \mathrm{~cm}$ depth. The mice were placed into the cylinders for $8 \mathrm{~min}$ and the immobility time of the last 6 min was determined. Immobility, in which the mice remained immobile or made only small limb movements necessary to float, were regarded as depression-like behavior. Following FST, 6 mice from each group were euthanized by cervical dislocation after each FST. Blood samples were collected by cardiac puncture for the determination of plasma montelukast concentration. The lungs were isolated and fixed in $10 \%$ formaldehyde solution for histopathological analysis (Fig. 1).

Histopathological analysis. Isolated lungs, following each FST were fixed in $10 \%$ formaldehyde at room temperature for $\geq 48$ h. Slides (thickness, $0.4 \mu \mathrm{m}$ ) were prepared from paraffin embedded sections and were stained with hematoxylin and eosin at room temperature for $1 \mathrm{~h}$ with an automated slide stainer (Thermo Shandon Varistain Gemini ${ }^{\mathrm{TM}}$; Thermo Fisher Scientific, Inc.). Histopathological analysis was performed for the OVA, control and montelukast-treated OVA groups to confirm the inflammation in the lungs following OVA and to examine whether there is a reduction in the inflammation following montelukast treatment. A total of 6 mice from each group were analyzed. Histopathologically, interalveolar (interstitial) thickening, peribronchial and perivascular inflammation were evaluated and each of these parameters were graded 


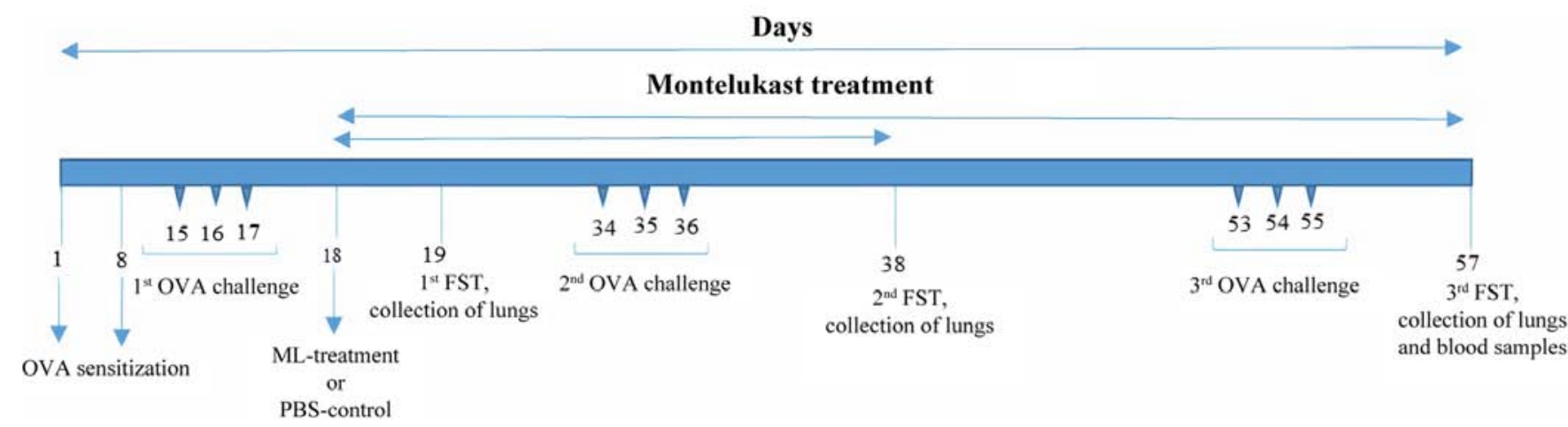

Figure 1. The experimental design for experimental asthma model, montelukast treatments and behavioral and histopathological analysis. OVA, ovalbumin; ML, montelukast; PBS, phosphate buffer saline; FST, forced swim test.

semiquantitatively in a blinded manner. The inflammation parameters were individually scored from $0-4$ ( 0 , no inflammation; 1 , mild inflammation; 2 , moderate inflammation; 3 , severe inflammation; 4, very severe inflammation) by evaluating the extent of inflammatory cells, tissue injury and interalveolar (interstitial) thickening (Leica Microsystems GmbH) Final scores were defined as the sum of peribronchial (0-4) and perivascular (0-4) inflammation.

Determination of plasma montelukast concentration. At the end of the experimental protocol, montelukast concentrations were confirmed in mice that had been treated with montelukast 40 days orally through drinking water. Blood samples were collected in heparinized tubes and centrifuged at $3.305 \mathrm{x} \mathrm{g}$ for $15 \mathrm{~min}$ to obtain plasma. The proteins in the plasma samples $(100 \mu \mathrm{l})$ were crushed with $100 \mu \mathrm{l}$ of acetonitrile (Sigma-Aldrich; Merck KGaA). The amount of montelukast in the plasma samples was quantified by liquid chromatography-electrospray ionization-mass spectrometry (LCMS-8030; Shimadzu Corporation). The mass spectrometric detection of montelukast was undertaken in the positive electrospray ionization and multiple reaction-monitoring mode using $586.0-422.10 \mathrm{~m} / \mathrm{z}$ transitions using $19 \mathrm{eV}$ of collision energy. The nebulizer gas flow and drying gas flow were $3 \mathrm{ml} / \mathrm{min}$ and $15 \mathrm{ml} / \mathrm{min}$, respectively. The heat blocking temperature, desolvation line temperature and collision-induced dissociation gas pressure were $400^{\circ} \mathrm{C}, 250^{\circ} \mathrm{C}$ and $15 \mathrm{kPa}$, respectively. Chromatographic separation was achieved on the $\mathrm{C}_{18}$ column (Hypersil ODS-4; GL Sciences; 50x2.1 mm; $3 \mu \mathrm{m}$ ) using a mobile phase consisting of acetonitrile containing $0.1 \%$ formic acid and water with $0.1 \%$ formic acid (70:30; v/v) at a flow rate of $0.3 \mathrm{ml} / \mathrm{min}$.

Open field tests. Open field tests were used to assess any changes in the locomotor activity of the mice following the administration of OVA or montelukast. The test was performed according to previous studies $(27,28)$. Briefly, black locomotor activity cages $(40 \times 40 \times 40 \mathrm{~cm})$ equipped with an infrared sensor system (Activity Meter; Commat, Ltd.) were used. The mice were individually placed in the center of the cages and tracked for $5 \mathrm{~min}$. The locomotor activities of the mice were assessed based on the total distance travelled in the cage as measured by the analyzing system. The test was performed in the OVA group 1 day after the completion of one OVA-challenge, a separate group that had been administered only montelukast ( $20 \mathrm{mg} / \mathrm{kg} /$ day) for 20 days and the control group.

Statistical analysis. Data are expressed as mean \pm standard error of the mean. Statistical analysis was performed using unpaired Student's t-test for in vivo assessment of airway function and one-way ANOVA followed by Tukey's post hoc test or the Kruskal Wallis test followed by Dunn's test for other experiments. Data were analyzed using GraphPad Prism software (version 5.0; GraphPad Software Inc.). P<0.05 was considered to indicate a statistically significant difference.

\section{Results}

Development and establishment of experimental asthma model. In the current study, the relationship between airway inflammation, chronic montelukast treatment and depression-like behavior was investigated. The induction of experimental asthma was confirmed by histopathological changes in lung segments and development of airway hyperresponsiveness to methacholine challenge. In order to maintain the allergic airway inflammation throughout the experimental protocol, the OVA-challenges were performed in triplicate as described in Fig. 1. This protocol allowed for the investigation of the effect of chronic allergic inflammation and chronic montelukast treatment on depression-like behavior. The occurrence of inflammation was presented as histopathological images (Fig. 2A and B). Total inflammation scores (Fig. 2C) and airway resistance (Fig. 2D) were significantly increased following the first OVA challenge in the OVA group compared with controls. Immobility time was significantly higher in the OVA group compared with controls (Fig. 2E). Furthermore, open field tests were performed to eliminate the possible effects of OVA and montelukast treatment on the locomotor activities of the mice. Total distances travelled in the cages in the OVA group were not significantly different compared with controls (Fig. 2F). Similarly, the montelukast-treated OVA group (20 days of treatment) did not exhibit a significant difference in total distances travelled, which was indicated the locomotor activity. Additionally, montelukast concentrations at a steady state were determined to be $226.40 \pm 34.59 \mathrm{ng} / \mathrm{ml}$ in mice administrated montelukast for $>40$ days (data not shown). 
A



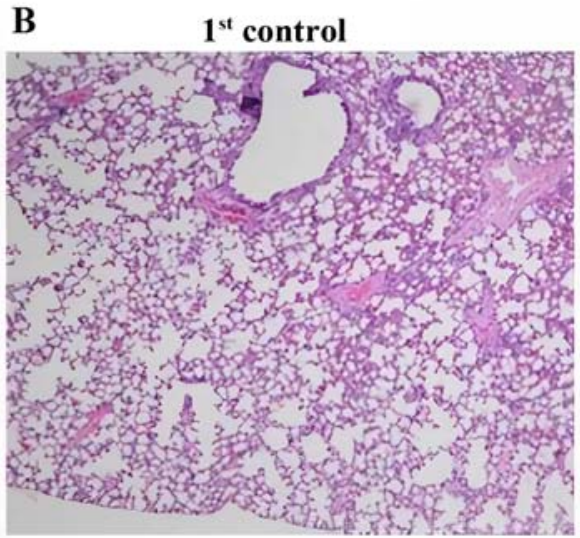

C
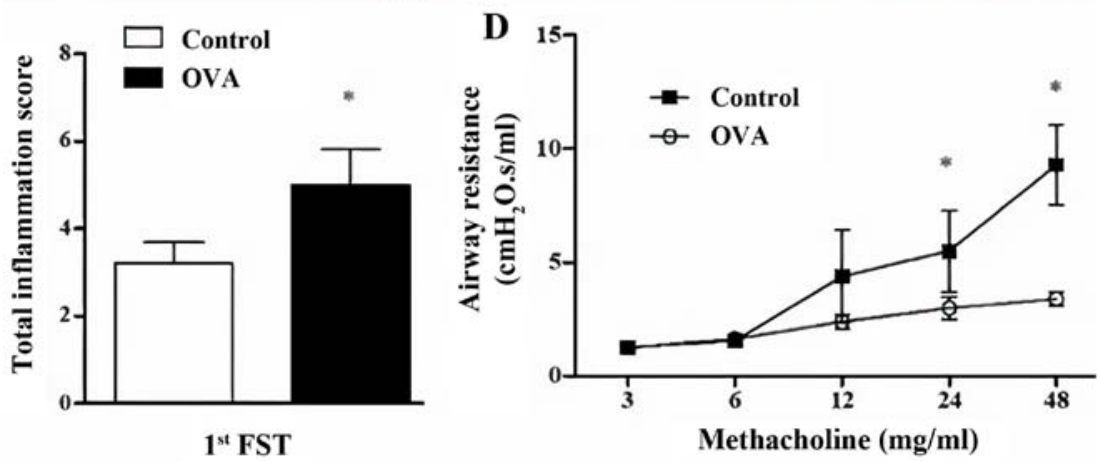

E

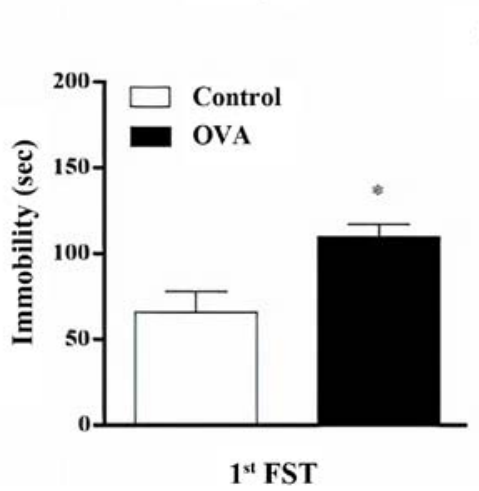

F

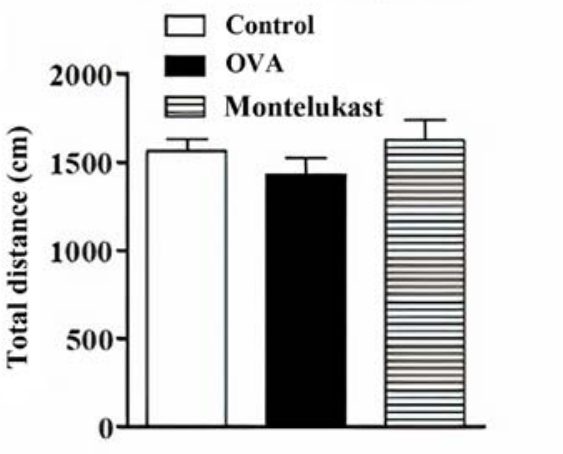

Figure 2. Histopathological images obtained from the lung samples of the (A) OVA and (B) control groups following the first OVA challenge. (C) Total inflammation scores of the lung tissues of the OVA and control groups ( $\mathrm{n}=6 /$ group). (D) Airway resistance was increased in the OVA-treated group compared with control group (after one OVA challenge; $n=8$ /group). (E) The immobility times of the OVA-treated and control mice following the first FST point (n=24/group). (F) Total distance travelled by the mice in the open field test ( $\mathrm{n}=10 /$ group). Data are presented as mean \pm standard error of the mean. * $<<0.05$ vs. controls. OVA, ovalbumin; FST, forced swim test.

Effect of experimental asthma and montelukast treatment in FST. Airway inflammation observed in the OVA group compared with control group was slightly reduced in the montelukast-treated OVA group (20 days of treatment) following the 2nd FST (Fig. 3A-C). The total inflammation score of the OVA group was statistically increased compared with the control group (Fig. 3D). However, the decrease in the total inflammation score of the montelukast-treated OVA group was not statistically significant compared with OVA group. Furthermore, there was no statistical differences between the montelukast-treated OVA and control groups.

FST was performed following each OVA challenge phase to examine the short- and long-term effects of asthma on depression-like behavior. Immobility times were significantly increased in the OVA group compared with the control group following the second challenge (Fig. 4). Furthermore, immobility times were significantly decreased in the montelukast-treated OVA group compared with the OVA group. Immobility times in the montelukast-treated control group were not significantly different between control and montelukast-treated OVA groups. Furthermore, the immobility times of montelukast-treated control group were significantly lower compared with the OVA group.

Following the third challenge, inflammation in OVA-treated lungs was reduced followed by ongoing montelukast treatment compared with the control group (5A-C) and total inflammation scores of the montelukast-treated OVA group were significantly decreased compared with the OVA group (Fig. 5D). Following the third challenge, chronic montelukast application to the OVA-treated group (40 days of treatment) significantly decreased immobility times 

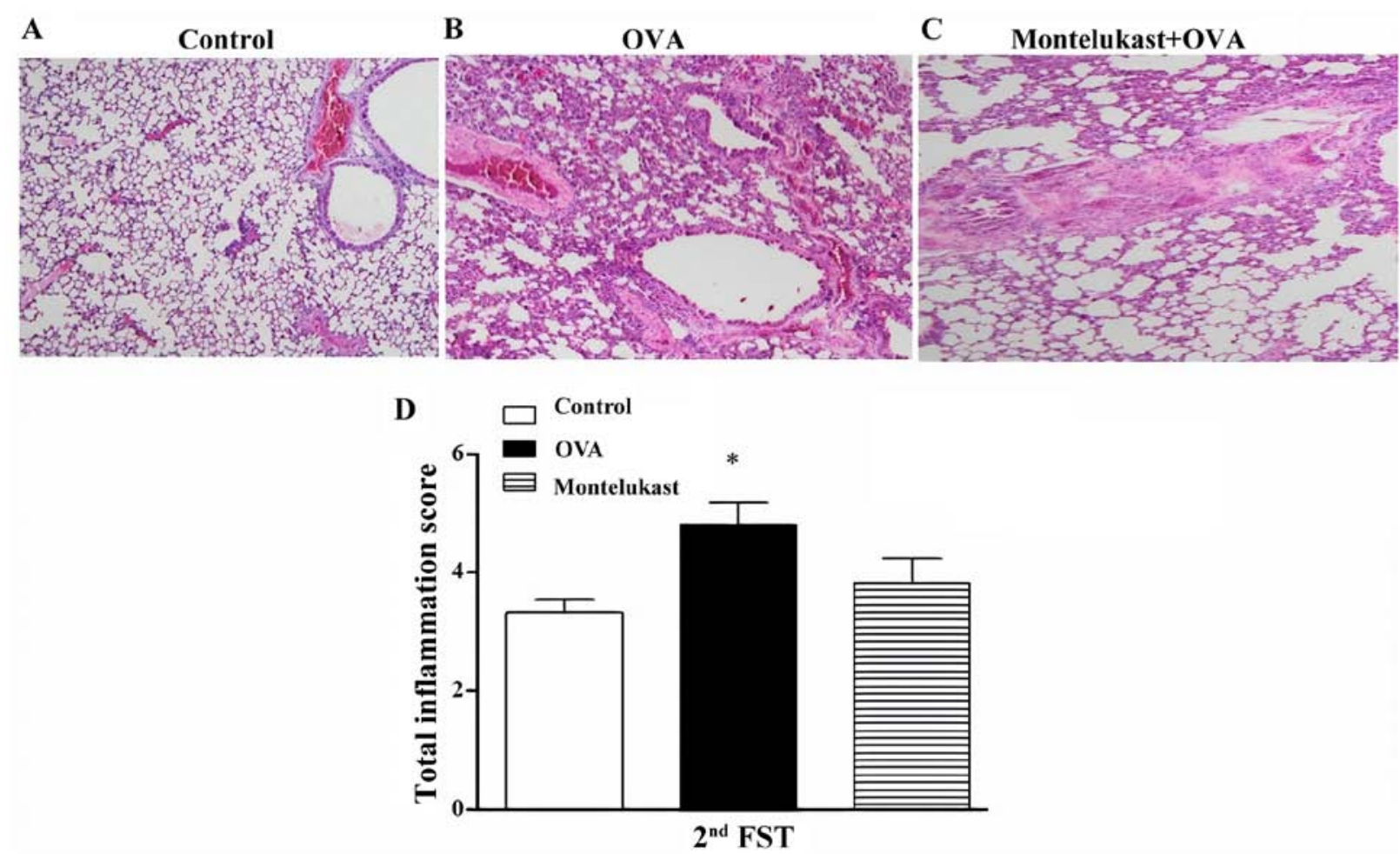

Figure 3. Histopathological images from the (A) control, (B) OVA and (C) montelukast-treated OVA (20 days of treatment) groups (n=6/group) following the second FST point (40x). (D) Total inflammation scores of the lung tissues of the control, OVA and montelukast-treated OVA groups ( $\mathrm{n}=6 / \mathrm{group}$ ). Data are presented as mean \pm standard error of the mean. ${ }^{*} \mathrm{P}<0.05$ vs. controls. OVA, ovalbumin; FST, force swim test.

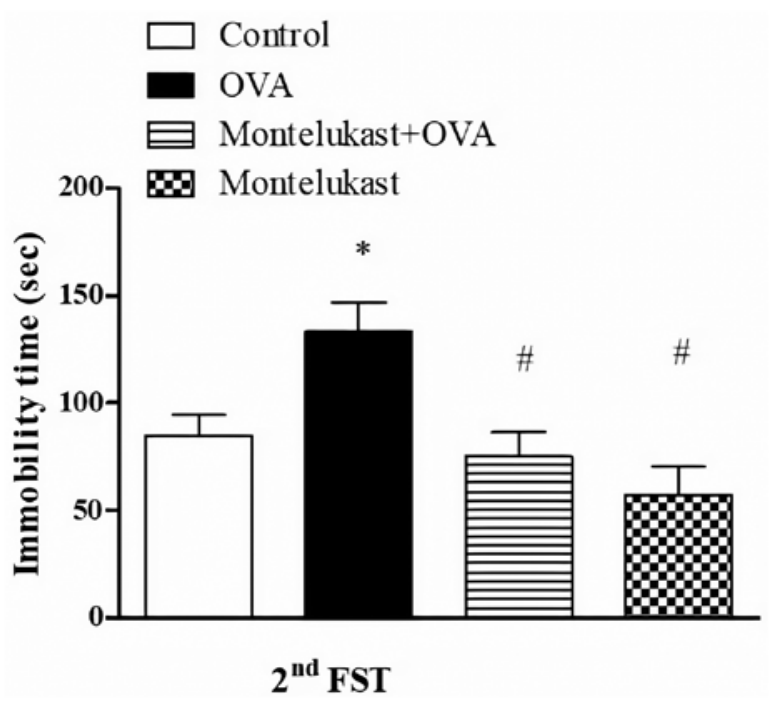

Figure 4. Immobility times of mice in the control, OVA, montelukast-treated OVA and montelukast-treated controls following the second FST point $(\mathrm{n}=18$ /group). Data are presented as mean \pm standard error of the mean. ${ }^{*} \mathrm{P}<0.05$ vs. controls. ${ }^{\#} \mathrm{P}<0.05$ vs. the OVA group. OVA, ovalbumin; FST, forced swim test.

compared with the OVA group (Fig. 6). Immobility times of the montelukast-treated control group were not statistically different compared with controls; however, they were significantly decreased compared with the OVA group (Fig. 6). Furthermore, there were no significant differences between the immobility times of three time points in the OVA-treated groups (data not shown).

\section{Discussion}

Montelukast is an imperative medication that is widely used and is recommended in the guidelines for the treatment of asthma $(6,29)$. In the present study, the depression-like behavioral effects of chronic montelukast administration were investigated in a murine model of experimental asthma. In accordance with the clinical use of montelukast, an OVA-induced asthma model was used to assess both the possible depression-like effects of montelukast treatment and asthma itself. The OVA model is well-established and is the most commonly used experimental asthma model (30), causing airway hyperreactivity, inflammatory cell infiltration and parenchymal inflammation (24).

The current study confirmed the development of experimental asthma with OVA by pathological assessments and OVA was demonstrated to result in a statistically significant increase in lung inflammation following the first challenge. Furthermore, the increase of airway resistance in response to methacholine challenge in the OVA-treated mice indicated that allergic asthma was successfully induced. These results confirmed changes in the lungs may have led to impaired respiratory function, which in turn, may have reduced the mobility of the mice. Furthermore, open field tests prior to FST were performed to eliminate any possible changes in motor activity that may affect the mice and cause false negative or positive results in FST. However, there were no significant difference in the total distance travelled by the mice between the OVA, montelukast-treated OVA and control groups. This indicated that differences observed in FST were not caused by motor disability, confirming the reliability of this test. 

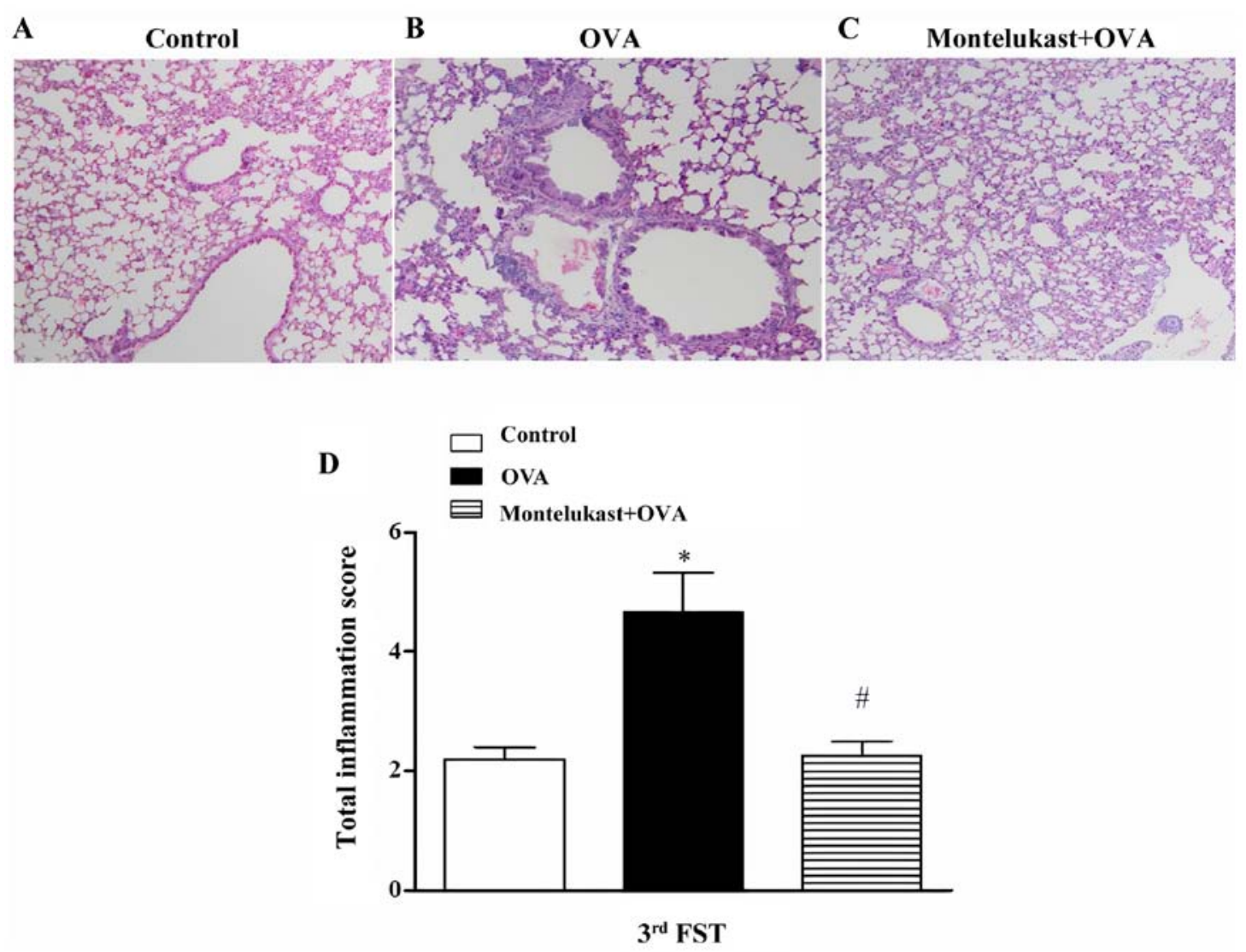

Figure 5. Histopathological images from the (A) control, (B) OVA and (C) montelukast-treated OVA groups following the third FST point (n=6/group; magnification, $\mathrm{x} 40$ ). (D) Total inflammation scores of the lung tissues of the control, OVA and montelukast-treated OVA groups ( $\mathrm{n}=6 / \mathrm{group})$. Data are presented as mean \pm standard error of the mean. ${ }^{*} \mathrm{P}<0.05$ vs. controls. ${ }^{*} \mathrm{P}<0.05$ vs. the OVA group. OVA, ovalbumin; FST, forced swim test.

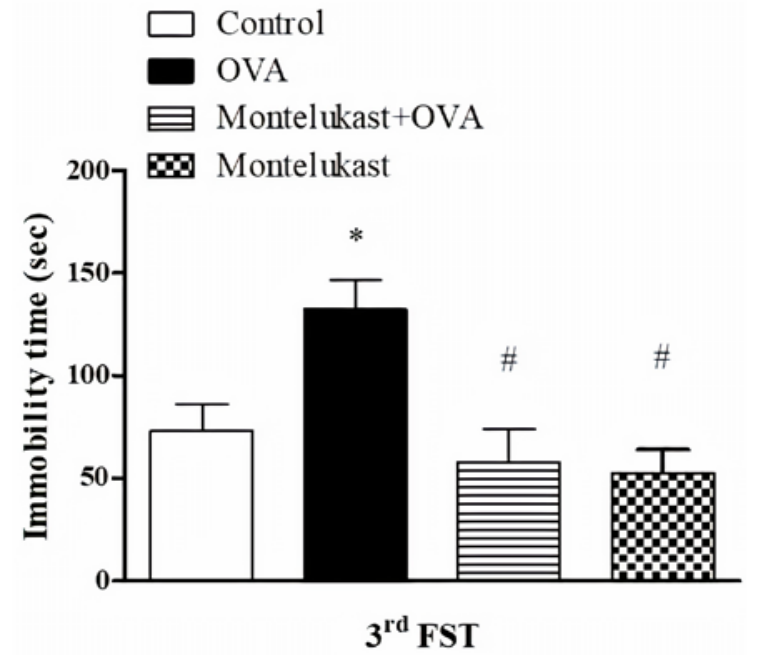

Figure 6. Immobility times of the mice in the control, OVA and montelukast-treated (40 days of treatment) groups ( $\mathrm{n}=12 /$ group). Data are presented as mean \pm standard error of the mean. ${ }^{*} \mathrm{P}<0.05$ vs. controls. ${ }^{~} \mathrm{P}<0.05$ vs. the OVA group. OVA, ovalbumin; FST, forced swim test.

Furthermore, the standard OVA protocol was modified to maintain the persistence of the disease and repeated challenges were applied to the mice at the indicated intervals. The modified model allowed for the evaluation of the relationship between ongoing respiratory tract inflammation, chronic montelukast treatment and depression-like behavior at different time points over two months. Montelukast was administrated to the mice via drinking water during this period to avoid the stress of daily gavage. The plasma montelukast concentrations of mice were verified at the end of the current study and concentrations were at therapeutic concentrations consistent with the plasma concentrations of humans and rats $(31,32)$.

The association between asthma and depression following each OVA-challenge point was investigated. For all three time points, the immobility of the OVA mice was significantly increased compared with controls. However, immobility times did not differ between time points in the OVA group. This result is consistent with the pathological analysis and indicated that the repeated OVA challenges did not aggravate the disease; instead, it maintained inflammation. Furthermore, the role of chronic montelukast treatment in depression was assessed with FST performed following the second and third OVA challenges. Montelukast treatment was found to ameliorate lung inflammation as confirmed by histopathological analysis. These results indicated that montelukast dose used in the present study was within the therapeutic range. Inflammation was reduced by 20 days of montelukast treatment and was completely reversed by the end of 40 days of treatment. Additionally, montelukast treatment reduced the immobility times of mice following both FST compared with the OVA group. This indicated that chronic montelukast treatment in asthma does not cause depression-like behavior. Furthermore, the reduced immobility times indicated that montelukast decreased asthma-induced depression by 
suppressing airway inflammation. Asthma has been demonstrated to be closely associated with depression (33-36). Since montelukast is most commonly used in the treatment of allergic asthma, the side effect of depression-like behavior caused by montelukast has been debated in terms of whether it is associated with the drug or the disease itself (9,33-36). Therefore, the effect of montelukast on the control mice was evaluated to test whether the drug caused depression-like behavior under physiological conditions. Montelukast application to the control mice did not cause any change in the immobility times following the second and third FST compared with controls at the same time points.

During FST, an increase in immobility time indicated behavioral despair and depression-like behavior $(26,37,38)$. FST is one of the oldest screening tests as it has been used for numerous years and is one of the most accepted models for the investigations of antidepressant activity $(30,39,40)$. Various drugs used in the treatment of depression were first assessed using this test $(30,40)$. However, this test has certain disadvantages. For instance, FST is weak at reflecting clinical situations. However, it is the most preferred pharmacological test for assessing antidepressant activity and will be beneficial in supporting future results using other methods, including the elevated plus maze or sucrose tolerance tests, which asses depressive-like behavior using different mechanisms (41).

In the current study, depression-like behavior was increased in asthmatic mice; however, there was no indicated association between montelukast treatment and depression. The association between allergic asthma and depression has been reported by several studies $(41,42)$. Numerous patients with chronic allergic asthma have a history of depression and patients with asthma are more susceptible to suicide $(43,44)$. Allergic asthma dependent-chronic stress may lead to depression or these two chronic diseases may coexist as they are affected by similar inflammatory pathways $(21,45)$. Increases in the levels of inflammatory mediators, including cytokines and interleukins have been demonstrated in depression and allergic asthma (21,45). Uz et al (46) indicated that in arachidonate 5-lipoxygenase knock-out animals, depression-like behavior declined due to a decrease in leukotrienes. Furthermore, previous studies have reported that the immobility time of mice and rats was increased by OVA-induced allergic asthma $(47,48)$; these results are consistent with the data of the current study. Pharmacokinetic studies have reported that the distribution of montelukast across the blood brain barrier is limited (12) and that the cysteinyl leukotriene receptor-1 is weakly expressed in brain tissue $(49,50)$. It has also been indicated that when montelukast treatment is not sufficient for allergic asthma therapy, mediators, including pro-inflammatory cytokines, increase due to unresolved asthma and cause sleep disorders, depression and suicidal behavior $(15,51)$.

Neuropsychiatric side effects were not reported in clinical trials and were not included in the prescribing information when Singulair ${ }^{\circledR}$ was first authorized. However, with the widespread use of montelukast, several post-marketing studies indicated the drug caused depression, hallucinations, sleep disorders and suicidal behavior (5). These side effects were reported following the beginning of treatment and were revealed to disappear following the cessation of the drug $(52,53)$. Some studies have not indicated an association between depression and montelukast treatment $(54,55)$. Furthermore, a previous study indicated that there was no difference in the side effect profiles of montelukast-treated and placebo groups (56). Clinical studies have several limitations in the assessment of behavioral adverse effects of drugs. In particular, the selection of patients for clinical studies serves a key role in determining the reliability of the research and it is challenging to exclude those who are susceptible to psychiatric diseases (57). Furthermore, diagnosis of depression primarily depends on the self-reports of patients, which can lead to abundant variation among study populations (57). Thus, contradictory results are expected in clinical studies on adverse behavioral effects (57).

The present experimental study had certain limitations, such as only using FST for assessing depression-like behavior. Although FST is a commonly used test for the evaluation of depressed activity, additional behavioral tests, including those for anxiety and hedonia, can be performed to confirm the side effects of montelukast.

In conclusion, montelukast is an efficient drug and is frequently the preferred treatment for asthma (9). Further experimental studies should be conducted to increase the reliability of clinical data, to achieve a better understanding of the mechanisms of neuropsychiatric side effects and to provide information for the safer use of montelukast.

\section{Acknowledgements}

The authors would like to thank Associate Professor Mikael Adner (Experimental Asthma and Allergy Research and The Centre for Allergy Research, Karolinska Institutet, Solna, Sweden) for his valuable contribution in developing the chronic OVA-induced murine asthma model and Dr Yasemin Karaman-Kutluay (Department of Pharmacy, Hacettepe University Faculty of Pharmacy, Ankara, Turkey) for her help in the in vivo assessment of airway function.

\section{Funding}

No funding was received.

\section{Availability of data and materials}

The datasets used and/or analyzed during the current study are available from the corresponding author on reasonable request.

\section{Authors' contributions}

BCT, TEB and GT conceived and designed the current study. BT and TEB conducted and GT performed the experiments. EN performed and evaluated analytical measurements. SO evaluated pathological data. GT analyzed data and wrote the original draft of the manuscript. BCT and TEB reviewed and edited the manuscript. All authors read and approved the final manuscript.

\section{Ethics approval and consent to participate}

Animal experiments were approved by the Animal Experimentations Local Ethics Board of Hacettepe University, Ankara, Turkey (approval nos. 2018/3-2 and 2020/3-13). 


\section{Patient consent for publication}

Not applicable.

\section{Competing interests}

The authors declare that they have no competing interests.

\section{References}

1. Harris K, Kneale D, Lasserson TJ, McDonald VM, Grigg J and Thomas J: School-based self-management interventions for asthma in children and adolescents: A mixed methods systematic review. Cochrane Database Syst Rev 1: CD011651, 2019.

2. Pawankar R, Canonica GW, Holgate ST and Lockey RF: Allergic diseases and asthma: A major global health concern. Curr Opin Allergy Clin Immunol 12: 39-41, 2012.

3. Woo LN, Guo WY, Wang X, Young A, Salehi S, Hin A, Zhang Y, Scott JA and Chow CW: A 4-week model of house dust mite (HDM) induced allergic airways inflammation with airway remodeling. Sci Rep 8: 6925, 2018.

4. Barnes PJ: New therapies for asthma: Is there any progress? Trends Pharmacol Sci 31: 335-343, 2010.

5. Calapai G, Casciaro M, Miroddi M, Calapai F, Navarra M and Gangemi S: Montelukast-induced adverse drug reactions: A review of case reports in the literature. Pharmacology 94: 60-70, 2014.

6. Haarman MG, van Hunsel F and de Vries TW: Adverse drug reactions of montelukast in children and adults. Pharmacol Res Perspect 5: e00341, 2017.

7. Aldea Perona A, Garcia-Saiz M and Sanz Alvarez E: Psychiatric disorders and montelukast in children: A disproportionality analysis of the VigiBase((R)). Drug Saf 39: 69-78, 2016.

8. Benard B, Bastien V, Vinet B, Yang R, Krajinovic M and Ducharme FM: Neuropsychiatric adverse drug reactions in children initiated on montelukast in real-life practice. Eur Respir J 50: 1700148, 2017.

9. https://ginasthma.org/wp-content/uploads/2020/04/GINA-2020full-report_-final-_wms.pdf.

10. FDA: Early Communication About an Ongoing Safety Review of Montelukast (Singulair). 3/23/2008 04/18/2013 8/7/2018]; Available from: https://wayback.archive-it org/7993/20170112033551/http:/www.fda.gov/Drugs/DrugSafety/ PostmarketDrugSafetyInformationforPatientsandProviders/ DrugSafetyInformationforHeathcareProfessionals/ucm070618.htm.

11. FDA: Updated Information on Leukotriene Inhibitors: Montelukast (marketed as Singulair), Zafirlukast (marketed as Accolate), and Zileuton (marketed as Zyflo and Zyflo CR). 8/28/2009 07/08/2015; https://wayback.archive-it. org/7993/20170111080414/http://www.fda.gov/Drugs/DrugSafety/ PostmarketDrugSafetyInformationforPatientsandProviders/ DrugSafetyInformationforHeathcareProfessionals/ucm165489.htm.

12. FDA. SINGULAIR ${ }^{\circledR}$ (Montelukast sodium) Tablets, Chewable Tablets, And Oral Granules 8/19/2009; Available from: https:// www.accessdata.fda.gov/drugsatfda_docs/label/2009/020829s0 51_020830s052_021409s028lbl.pdf.

13. Manalai P, Woo JM and Postolache TT: Suicidality and montelukast. Expert Opin Drug Saf 8: 273-282, 2009.

14. Schumock GT, Stayner LT, Valuck RJ, Joo MJ, Gibbons RD and Lee TA: Risk of suicide attempt in asthmatic children and young adults prescribed leukotriene-modifying agents: A nested case-control study. J Allergy Clin Immunol 130: 368-375, 2012.

15. Postolache TT, Komarow H and Tonelli LH: Allergy: A risk factor for suicide? Curr Treat Options Neurol 10: 363-376, 2008

16. Hurwitz EL and Morgenstern H: Cross-sectional associations of asthma, hay fever, and other allergies with major depression and low-back pain among adults aged 20-39 years in the United States. Am J Epidemiol 150: 1107-1116, 1999.

17. Addolorato G, Ancona C, Capristo E, Graziosetto R, Di Rienzo L, Maurizi $\mathrm{M}$ and Gasbarrini G: State and trait anxiety in women affected by allergic and vasomotor rhinitis. J Psychosom Res 46 : 283-289, 1999.

18. Trojan TD, Khan DA, Defina LF, Akpotaire O, Goodwin RD and Brown ES: Asthma and depression: The cooper center longitudinal study. Ann Allergy Asthma Immunol 112: 432-436, 2014 .
19. Oraka E, King ME and Callahan DB: Asthma and serious psychological distress: Prevalence and risk factors among US adults, 2001-2007. Chest 137: 609-616, 2010.

20. Wong KO, Rowe BH, Douwes J and Senthilselvan A: Asthma and wheezing are associated with depression and anxiety in adults: An analysis from 54 countries. Pulm Med 2013: 929028 , 2013.

21. Jiang M, Qin P and Yang X: Comorbidity between depression and asthma via immune-inflammatory pathways: A meta-analysis. J Affect Disord 166: 22-29, 2014.

22. Katon W, Lozano P, Russo J, McCauley E, Richardson L and Bush T: The prevalence of DSM-IV anxiety and depressive disorders in youth with asthma compared with controls. J Adolesc Health 41: 455-463, 2007.

23. eurlex.europa.eu/LexUriServ/LexUriServ.do?uri=OJ:L:2010:276 :0033:0079:En:PDF

24. Swedin L, Ellis R, Kemi C, Ryrfeldt A, Inman M, Dahlén SE and Adner M: Comparison of aerosol and intranasal challenge in a mouse model of allergic airway inflammation and hyperresponsiveness. Int Arch Allergy Immunol 153: 249-258, 2010.

25. Porsolt RD, Bertin A and Jalfre M: Behavioral despair in mice: A primary screening test for antidepressants. Arch Int Pharmacodyn Ther 229: 327-336, 1977.

26. Porsolt RD, Le Pichon M and Jalfre M: Depression: A new animal model sensitive to antidepressant treatments. Nature 266: 730-732, 1977.

27. Painsipp E, Wultsch T, Edelsbrunner ME, Tasan RO, Singewald N, Herzog H and Holzer P: Reduced anxiety-like and depression-related behavior in neuropeptide Y Y4 receptor knockout mice. Genes Brain Behav 7: 532-542, 2008.

28. Brunner SM, Farzi A, Locker F, Holub BS, Drexel M, Reichmann F, Lang AA, Mayr JA, Vilches JJ, Navarro X, et al: GAL3 receptor KO mice exhibit an anxiety-like phenotype. Proc Natl Acad Sci USA 111: 7138-7143, 2014.

29. British Thoracic Society; Scottish Intercollegiate Guidelines Network: British guideline on the management of asthma. Thorax 69 (Suppl 1): 1-192, 2014.

30. Aun MV, Bonamichi-Santos R, Arantes-Costa FM, Kalil J and Giavina-Bianchi P: Animal models of asthma: Utility and limitations. J Asthma Allergy 10: 293-301, 2017.

31. Zhao JJ, Rogers JD, Holland SD, Larson P, Amin RD, Haesen R, Freeman A, Seiberling M, Merz M and Cheng H: Pharmacokinetics and bioavailability of montelukast sodium (MK-0476) in healthy young and elderly volunteers. Biopharm Drug Dispos 18: 769-777, 1997.

32. Marschallinger J, Krampert M, Couillard-Despres S, Heuchel R, Bogdahn U and Aigner L: Age-dependent and differential effects of Smad7DEx1 on neural progenitor cell proliferation and on neurogenesis. Exp Gerontol 57: 149-154, 2014.

33. Brunner WM, Schreiner PJ, Sood A and Jacobs DR Jr: Depression and risk of incident asthma in adults. The CARDIA study. Am J Respir Crit Care Med 189: 1044-1051, 2014.

34. de Miguel Diez J, Hernández Barrera V, Puente Maestu L, Carrasco Garrido P, Gómez García T and Jiménez García R: Psychiatric comorbidity in asthma patients. Associated factors. J Asthma 48: 253-258, 2011.

35. Di Marco F, Santus P and Centanni S: Anxiety and depression in asthma. Curr Opin Pulm Med 17: 39-44, 2011.

36. Gerald JK and Moreno FA: Asthma and depression: It's complicated. J Allergy Clin Immunol Pract 4: 74-75, 2016.

37. Petit-Demouliere B, Chenu F and Bourin M: Forced swimming test in mice: A review of antidepressant activity. Psychopharmacology (Berl) 177: 245-255, 2005.

38. Kara NZ, Stukalin Y and Einat H: Revisiting the validity of the mouse forced swim test: Systematic review and meta-analysis of the effects of prototypic antidepressants. Neurosci Biobehav Rev 84: 1-11, 2018.

39. Slattery DA and Cryan JF: Using the rat forced swim test to assess antidepressant-like activity in rodents. Nat Protoc 7: 1009-1014, 2012

40. Slattery DA and Cryan JF: The ups and downs of modelling mood disorders in rodents. ILAR J 55: 297-309, 2014.

41. Van Lieshout RJ, Bienenstock J and MacQueen GM: A review of candidate pathways underlying the association between asthma and major depressive disorder. Psychosom Med 71: 187-195, 2009.

42. Gao YH, Zhao HS, Zhang FR, Gao Y, Shen P, Chen RC and Zhang GJ: The relationship between depression and asthma: A meta-analysis of prospective studies. PLoS One 10: e0132424, 2015. 
43. Postolache TT, Langenberg P, Zimmerman SA, Lapidus M Komarow H, McDonald JS, Furst N, Dzhanashvili N, Scrandis D, Bai J, et al: Changes in severity of allergy and anxiety symptoms are positively correlated in patients with recurrent mood disorders who are exposed to seasonal peaks of aeroallergens. Int $\mathbf{J}$ Child Health Hum Dev 1: 313-322, 2008.

44. Goodwin RD and Eaton WW: Asthma, suicidal ideation, and suicide attempts: Findings from the Baltimore epidemiologic catchment area follow-up. Am J Public Health 95: 717-722, 2005.

45. Loerbroks A, Gadinger MC, Bosch JA, Stürmer T and Amelang M: Work-related stress, inability to relax after work and risk of adult asthma: A population-based cohort study. Allergy 65: 1298-1305, 2010

46. Uz T, Dwivedi Y, Pandey GN, Roberts RC, Conley RR, Manev R and Manev H: 5-Lipoxygenase in the prefrontal cortex of suicide victims. Open Neuropsychopharmacol J 1: 1-5, 2008.

47. Zuo HX, Li JQ, Han B, Ke CJ, Liu XD, Zhang YC, Li L and Yang X: Di-(n-butyl)-phthalate-induced oxidative stress and depression-like behavior in mice with or without ovalbumin immunization. Biomed Environ Sci 27: 268-280, 2014.

48. Neamati A, Chaman F, Hosseini M and Boskabady MH: The effects of Valeriana officinalis L. hydro-alcoholic extract on depression like behavior in ovalbumin sensitized rats. J Pharm Bioallied Sci 6: 97-103, 2014

49. Lynch KR, O'Neill GP, Liu Q, Im DS, Sawyer N, Metters KM Coulombe N, Abramovitz M, Figueroa DJ, Zeng Z, et al: Characterization of the human cysteinyl leukotriene CysLT1 receptor. Nature 399: 789-793, 1999.

50. Jones TR and Rodger IW: Role of leukotrienes and leukotriene receptor antagonists in asthma. Pulm Pharmacol Ther 12: $107-110,1999$.
51. de Vries TW and van Hunsel F: Adverse drug reactions of systemic antihistamines in children in the netherlands. Arch Dis Child 101: 968-970, 2016.

52. Callero-Viera A, Infante S, Fuentes-Aparicio V, Zapatero L and Alonso-Lebrero E: Neuropsychiatric reactions to montelukast. J Investig Allergol Clin Immunol 22: 452-453, 2012.

53. Alkhuja S, Gazizov N and Alexander ME: Sleeptalking! Sleepwalking! Side effects of montelukast. Case Rep Pulmonol 2013: 813786, 2013.

54. Ali MM, O'Brien CE, Cleves MA and Martin BC: Exploring the possible association between montelukast and neuropsychiatric events among children with asthma: A matched nested case-control study. Pharmacoepidemiol Drug Saf 24: 435-445, 2015.

55. Holbrook JT and Harik-Khan R: Montelukast and emotional well-being as a marker for depression: Results from 3 randomized, double-masked clinical trials. J Allergy Clin Immunol 122: 828-829, 2008

56. Philip G, Hustad C, Noonan G, Malice MP, Ezekowitz A, Reiss TF and Knorr B: Reports of suicidality in clinical trials of montelukast. J Allergy Clin Immunol 124: 691-696 e6, 2009.

57. Philip G, Hustad CM, Malice MP, Noonan G, Ezekowitz A, Reiss TF and Knorr B: Analysis of behavior-related adverse experiences in clinical trials of montelukast. J Allergy Clin Immunol 124: 699-706 e8, 2009.

This work is licensed under a Creative Commons Attribution-NonCommercial-NoDerivatives 4.0 International (CC BY-NC-ND 4.0) License. 